\title{
Conservation of the deadenylase activity of proteins of the Caf1 family in human
}

\author{
CLAIRE BIANCHIN, ${ }^{1}$ FABIENNE MAUXION, ${ }^{2}$ STÉPHANIE SENTIS, ${ }^{1}$ BERTRAND SÉRAPHIN, ${ }^{2}$ and \\ LAURA CORBO' \\ ${ }^{1}$ Unité INSERM U590, Centre Léon Bérard, 69373 Lyon Cedex 08, France \\ ${ }^{2}$ Equipe Labellisée La Ligue, Centre de Génétique Moléculaire, CNRS UPR2167 Associée à l'Université Pierre et Marie Curie, \\ 91198 Gif sur Yvette, France
}

\begin{abstract}
The yeast Pop2 protein, belonging to the eukaryotic Caf1 family, is required for mRNA deadenylation in vivo. It also catalyzes poly(A) degradation in vitro, even though this property has been questioned. Caf1 proteins are related to RNase D, a feature supported by the recently published structure of Pop2. Yeast Pop2 contains, however, a divergent active site while its human homologs harbor consensus catalytic residues. Given these differences, we tested whether its deadenylase activity is conserved in the human homologs Caf1 and Pop2. Our data demonstrate that both human factors degrade poly(A) tails indicating their involvement in mRNA metabolism.
\end{abstract}

Keywords: Ccr4, mRNA decay, poly(A), Caf1, Pop2, RNase D

\section{INTRODUCTION}

The control of gene expression occurs at each of the steps leading to protein synthesis including transcription, mRNA processing, translation, and mRNA decay. Analyses of eukaryotic mRNA decay, mainly performed in yeast, have shown that this process is initiated by deadenylation (except in the case of the degradation of aberrant mRNAs) (Parker and Song 2004). During deadenylation, the size of the poly $(\mathrm{A})$ tail of the target mRNA is reduced to $10-15$ residues (Muhlrad et al. 1994). This event triggers the degradation of the mRNA body by various exonucleases (Muhlrad et al. 1994) while the mRNA cap is catabolized by various decapping enzymes (Wang and Kiledjian 2001; LykkeAndersen 2002; van Dijk et al. 2002, 2003; Wang et al. 2002). Interestingly, the speed of the deadenylation process has been shown to vary widely between mRNAs and degradation of the poly $(\mathrm{A})$ tail appears to be a rate-limiting step in the decay process (Muhlrad et al. 1995). Identifying enzymes implicated in poly $(\mathrm{A})$ tail removal is therefore of

Reprint requests to: Bertrand Séraphin, Equipe Labellisée La Ligue, Centre de Génétique Moléculaire, CNRS UPR2167 Associée à l'Université Pierre et Marie Curie, 91198 Gif sur Yvette, France; e-mail: seraphin@cgm.cnrs-gif.fr; fax: 331698238 77; or Laura Corbo, Unité INSERM U590, Centre Léon Bérard, 28, Rue Laënnec, 69373 Lyon Cedex 08, France; e-mail: corbo@lyon.fnclcc.fr; fax: 33478782720.

Article and publication are at http://www.rnajournal.org/cgi/doi/ 10.1261/rna.7135305. prime interest, as these are likely to be the targets of regulators affecting mRNA stability.

The first deadenylase identified was purified from yeast and shown to degrade poly(A) tails bound to poly(A) binding protein (Boeck et al. 1996). This enzyme is heterodimeric, one subunit, Pan2, harboring the catalytic site while the role of the second subunit, Pan3, remains unclear even though it is absolutely required for activity (Brown et al. 1996). Surprisingly, however, deletion of genes encoding Pan 2 or Pan 3 did not generate a clear growth phenotype. It did not strongly affect mRNA degradation in vivo either. These results suggested that other deadenylase(s) were encoded in the yeast genome. A second deadenylase was purified from calf thymus based on its activity and named PARN for $\underline{P}$ oly $(\underline{A})$-specific RiboNuclease (Korner et al. 1998). In contrast to Pan2/Pan3, PARN was inhibited by poly(A) binding protein. Interestingly, however, PARN is stimulated by the presence of a cap at the $5^{\prime}$ end of the substrate mRNA (Dehlin et al. 2000; Gao et al. 2000). Phylogenetic analyses demonstrated that PARN is present in mammals but absent from several organisms including yeast and fungi. Interestingly, both Pan 2 and PARN where shown to be related to the bacterial RNAse D protein, which is involved in the exonucleolytic digestion of pre-tRNA $3^{\prime}$ extensions. Searches for yeast proteins similar to RNase D led to the identification of yeast Pop2 as a putative deadenylase (Daugeron et al. 2001). This protein had been origi- 
nally implicated in the control of gene expression and proposed to act at the level of transcription (Denis and Chen 2003). The latter suggestion has, however, never been confirmed by direct run-on experiments, and several of the original observations could as well be reinterpreted as resulting from an implication of Pop2 in mRNA degradation. Pop2 belongs to a large family of eucaryotic proteins named Caf1. This family is one of the member of the DEDD superfamily of $3^{\prime}-5^{\prime}$ exonucleases that encompasses both RNase and Dnase activities. These proteins are characterized by the presence of four conserved acidic amino acids, three aspartates (D) and one glutamate (E), that are located in three conserved regions of the protein in the order DEDD. These residues coordinate two magnesium ions involved in the catalysis. A fifth residue, histidine or tyrosine, located before the last conserved $\mathrm{D}$, is also highly conserved but its function is not entirely clear. Pop2 is, however a divergent member of the family because of the presence of a nonconserved large N-terminal extension, and because it does not contain consensus residues at two of the active site positions involved in coordinating the magnesium ions, the consensus DEDD being changed to SEDQ (with S-serine and Q-glutamine). In addition, the conserved histidine/tyrosine has been substituted by a threonine (Daugeron et al. 2001; Thore et al. 2003) (Fig. 1). These properties are conserved among several yeast species but not in other branches of the eukaryotic lineage. This suggested either that Pop2 had lost its catalytic activity or that the active site was able to accommodate alternative configurations while remaining functional. The latter turned out to be the case, as a recombinant Pop2 protein fragment lacking the yeast specific Nterminal extension was shown to display nuclease activity in vitro (Daugeron et al. 2001; Thore et al. 2003). Interestingly, Pop2 had been shown to associate with the Ccr4 protein, which also contained a putative nuclease domain (Dlakic 2000), thus supporting further a role in RNA turnover. Analysis of mRNA decay in yeast strains devoid of Pop2 or Ccr4 demonstrated that both proteins are required for deadenylation as well as normal mRNA degradation in vivo (Daugeron et al. 2001; Tucker et al. 2001). However, the respective role of Pop2 and Ccr4 in this process could not be discriminated in these original experiments. This ambiguity was resolved by subsequent analyses with point mutants, which revealed a major role for Ccr4 in the catalysis of deadenylation (Chen et al. 2002; Tucker et al. 2002). By contrast, point mutation abolishing the in vitro nuclease activity of Pop2 had little effect on mRNA poly(A) length in vivo. Taken together with the fact that another group was unable to detect deadenylation activity with a full-length recombinant Pop2, this led to question its activity (Chen et al. 2002; Tucker et al. 2002). However, determination of the structure of the conserved domain of Pop2 demonstrated that, despite its divergence, its fold is related to the superfamily of DEDD nucleases (Thore et al. 2003). Furthermore, phylogenetic analyses taken together with this structure indicated that the most conserved region of the protein is its catalytic site. This is a strong argument supporting the nuclease activity of Pop2 and its implication in mRNA decay. To support further this conclusion, we were interested to learn whether canonical members of the Caf1 family harboring

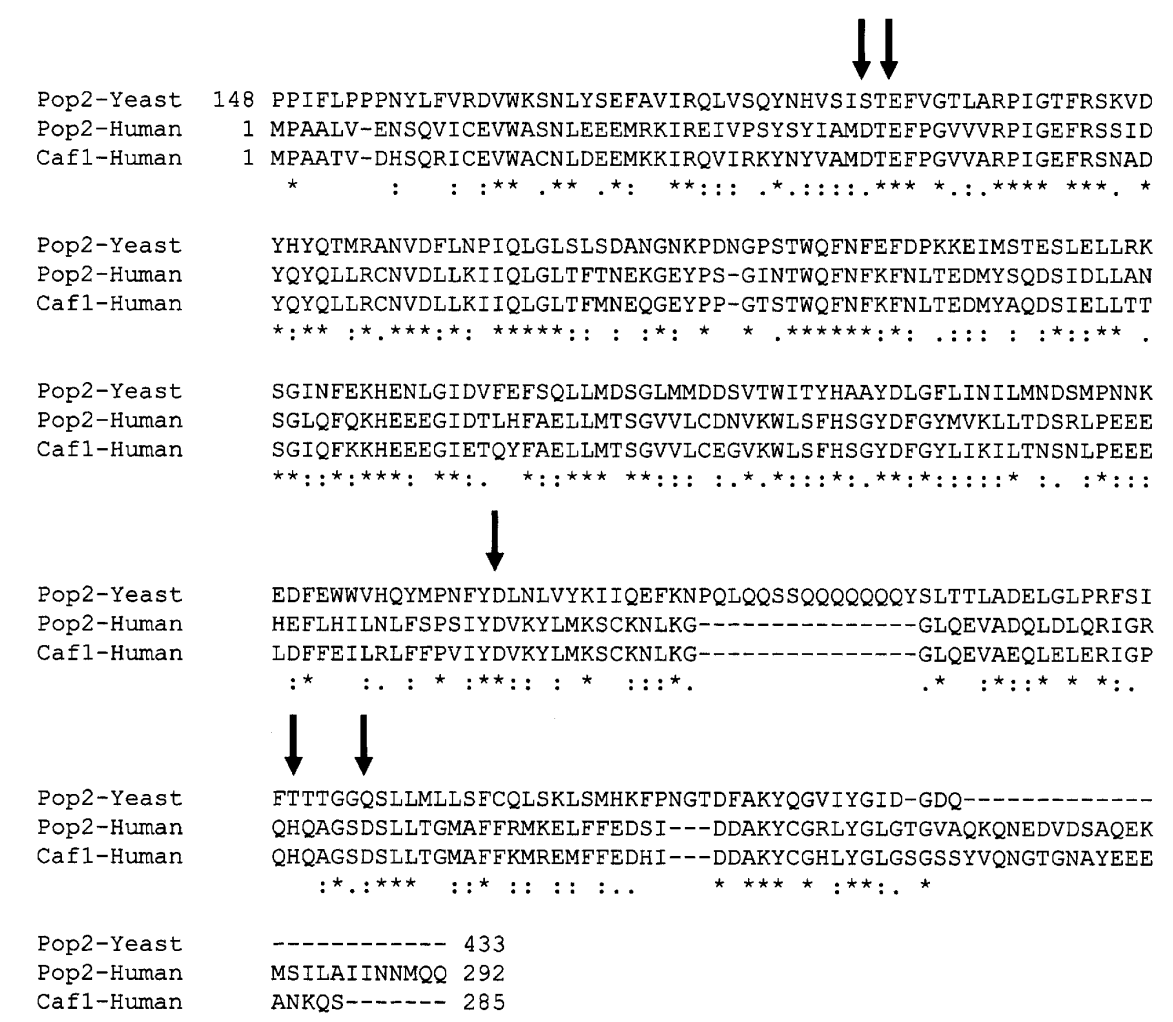

FIGURE 1. Alignment of the sequences of yeast Pop2, human Pop2, and human Caf1. Sequences were aligned with ClustalX (Thompson et al. 1997) and the resulting alignment manually edited. Sequence conservation is indicated below the aligned sequences with stars $\left.{ }^{*}\right)$ indicating complete amino-acid conservation between the three sequences while semicolon $(:)$ and dot (.) denote the presence of amino acids of high or low similarity, respectively. Conserved residues involved in magnesium binding and nuclease function in the RNase D subfamily of DEDD nucleases are indicated by arrows above the sequence. Amino acids at these first two positions were changed to Alanine by site-directed mutagenesis in the inactive human Caf1 mutant. The numbering, in the sequence, of the first and last aligned residues are indicated. Note that yeast Pop2 contains a large $\mathrm{N}$-terminal extension of 148 residues compared to its human counterparts, and that it diverges in the first and last residues involved in nuclease function. 
consensus catalytic site residues were also active in mRNA deadenylation. We report here that recombinant hCafl and hPop2 are enzymatically active, even though some interesting differences, can be found between the activities of these two factors. These results indicate that the deadenylase activity of yeast Pop2 is evolutionarily conserved in the Caf1 family, which is thus implicated in the control of gene expression by affecting mRNA decay.

\section{RESULTS}

To test whether human Caf1 (hCaf1) is able to degrade poly $(\mathrm{A})$, we prepared a recombinant form of the protein by overexpressing a GST-hCaf1 fusion in Escherichia coli. The protein was purified to apparent homogeneity by affinity on glutathione agarose (Fig. 2A). To test whether the recombinant protein is active, we prepared radiolabeled poly $(\mathrm{A})$ by extending cold poly $(\mathrm{A})$ with poly $(\mathrm{A})$ polymerase. GSThCaf1 was shown to degrade this substrate through the release of soluble material after TCA precipitation (data not show). This assay is, however, difficult to quantify because of the size and radiolabeling heterogeneity of the substrate. To assess more thoroughly the enzyme activity, recombinant hCaf1 was incubated with a $5^{\prime}$ end-labeled 14 nucleotide long synthetic RNA oligonucleotide (RNA7A) ending with seven A residues at its $3^{\prime}$. Time-course reactions were performed with various quantities of the recombinant factor. Reaction products were resolved on polyacrylamide gels and detected by autoradiography. These analyses demonstrated that the recombinant hCafl protein was able to de- grade the substrate RNA, as shorter products absent from the starting substrates appeared with time (Fig. 2B). The specific activity of the enzyme was poor, possibly due to poor folding in the presence of its natural partners. The stepwise shortening of the $5^{\prime}$-end labeled substrate RNA indicates that RNA degradation proceeds exonucleolytically in the $3^{\prime}$ to $5^{\prime}$ direction. Incubation of the enzyme with a molar excess of substrate revealed the simultaneous presence of a low level of fully degraded product when a large fraction of the substrate remained intact (see supplementary data S1). This suggests but does not prove that the enzyme is processive. Further detailed studies will be required to estimate more precisely the enzyme processivity. Interestingly, the enzyme was not completely blocked when it encountered the first non-A residue, a $U$ in this specific case, as shorter products were apparent. However, the accumulation of a product resulting from the degradation of the first seven A residues, particularly visible at high enzyme concentration because a lower protein amount was not allowed to reach this point, suggests that the enzyme was slowed down when it encountered the $U$ residue. This observation is consistent with a preference or specificity for A nucleotide. Interestingly, once the $U$ residue was removed, the enzyme was able to degrade the three following A residues. No further degradation was visible. Other substrates were used to ascertain the general validity of this result. Results obtained with a 27-nt long ribo-oligonucleotide substrate harboring five A residues at its $3^{\prime}$ end (RNA5A) are shown in Figure 2C. Again, exonucleolytic degradation of the substrate was detected. Interestingly, in this case, the enzyme was not able to bypass the first non-A residues,
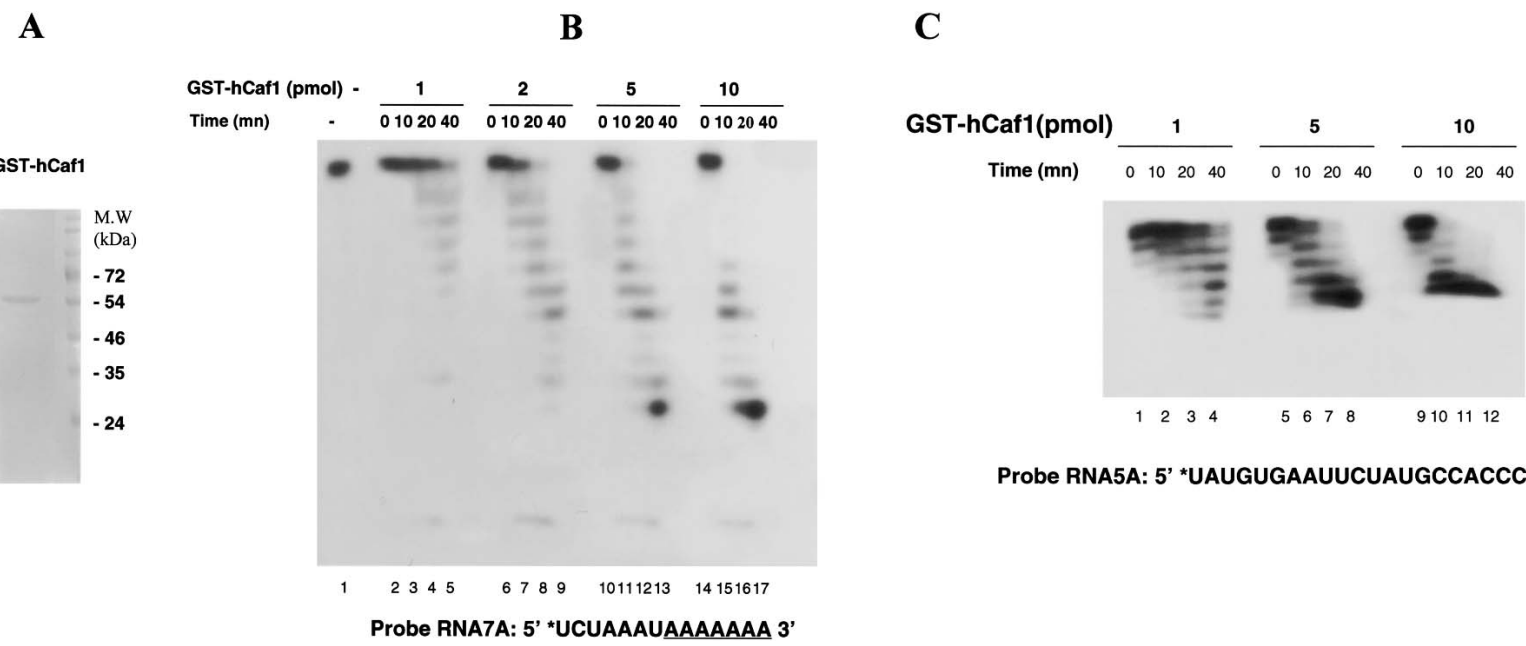

Probe RNA5A: 5' *UAUgugaAuUCuAUGCCACCCCAAAAA 3'

FIGURE 2. The recombinant GST-Caf1 protein is a deadenylase. (A) Protein profile of the recombinant GST-hCaf1 fraction. A Coomassie stained gel of a typical purified GST-hCaf1 fraction is shown. The migration of a molecular weight size marker is indicated. $(B)$ GST-hCaf1 is a RNAse. Gel-fractionated products of an RNA degradation assay using a 5' end-labeled synthetic RNA oligonucleotide are shown. Various quantities of the recombinant protein were used in parallel time-course reactions; $60 \mathrm{fmol}$ of substrate were used per reaction. Lane 1 shows the starting substrate. GST-hCaf1 pauses after degradation of the first seven A residues but resumes once the first U residue has been removed. (C) GST-hCaf1 specifically removes $3^{\prime}$ terminal A residues. Degradation of a 5'-labeled substrate ending by 5 A residues stops once the $3^{\prime}$ oligo(A) tail has been degraded. 
indicating a strong specificity for A nucleotides. Additional substrates were tested including in vitro transcribed RNAs and/or synthetic oligonucleotides containing longer $3^{\prime} \mathrm{A}$ tracks (Fig. 4A, lanes 1-6). Results consistent to the ones described above were obtained, indicating that these observations were not biased by the exact sequence and/or length of the RNA (data not shown). In addition, these results indicated that the structure of the RNA 5' end (mono- or tri-phosphate) did not affect degradation by Caf1. Finally, increased salt concentration up to $100 \mathrm{mM} \mathrm{KCl}$ in the assay did not affect the specificity of the enzyme even though it did slow down the degradation reaction (see supplementary data S2).

The results presented above suggest that hCaf1 is catalytically active. The specific exonucleolytic degradation of $3^{\prime}$ oligo(A) tail was inconsistent with the activity emanating from a contaminating nuclease from E. coli or from contaminants (e.g., RNase A) introduced during recombinant protein preparation. Two additional experiments were performed to definitively exclude this possibility. First, GST purified under strictly the same conditions was used to replace Caf1 in degradation experiments. No degradation of the substrates RNA was detected (Fig. 3, lanes 1-16). We also changed by site-directed mutagenesis two catalytic residues coordinating magnesium, namely the aspartate at position 40 and the glutamate at position 42 , to alanine to inactivate the protein. The mutant protein was purified in the same conditions as the wild type and tested for its ability

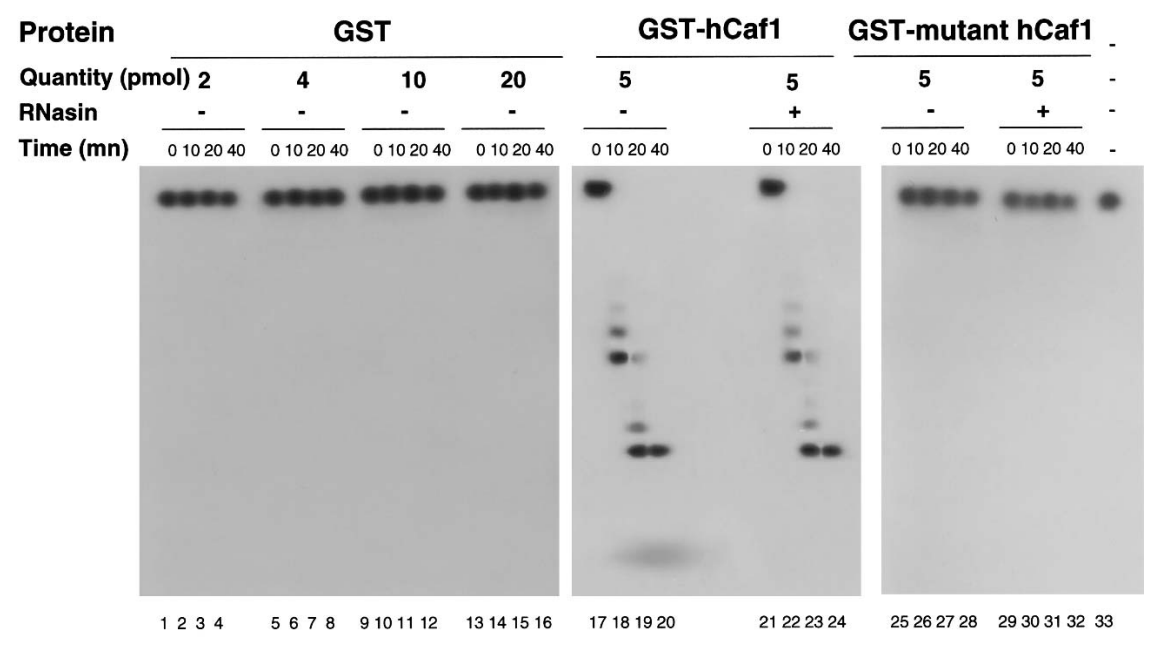

Probe RNA 7A: 5' *UCUAAAUAAAAAAA 3'

FIGURE 3. The deadenylase activity of hCafl does not result from the presence of contaminants. The deadenylase activity of recombinant GST-hCaf1 is specific. Time-course reaction was performed with the substrate ending with seven A residues and the indicated quantities of various proteins; $60 \mathrm{fmol}$ of substrate were used per reaction. Lanes 1-16: GST preparations purified in parallel with GST-hCafl do not display deadenylase activity, ruling out the copurification of contaminating nucleases from E. coli. The deadenylase activity observed with GST-hCaf1 is not inhibited by RNasin (cf. lanes 17-20 and lanes 21-24) excluding contamination of the recombinant fraction by common RNAse. Finally, a recombinant catalytic site mutant is unable to degrade RNA (lanes 25-32). The free substrate is shown in lane 33. Overall, these results demonstrate unequivocally that GST-hCaf1 is responsible for the deadenylase activity detected. to degrade substrate RNAs. Again, no degradation of the substrate mRNA was detected (Fig. 3, lanes 25-32). Furthermore, addition of RNAsin, an inhibitor of mainly commonly encountered contaminating nucleases, did not inhibit the activity associated with wild-type hCaf1 in standard degradation reactions (Fig. 3, cf. lanes 17-20 and 2124). Taken together, these various results indicate that Caf1 is an active deadenylase that is responsible for the

We next addressed the specificity of hCaf1. We tested the ability of hCaf1 to degrade RNA ending with non-A residues at their $3^{\prime}$ end or to digest internal poly(A) track. For this purpose, a $5^{\prime}$-labeled RNA oligonucleotide containing seven residues followed by 10 As and 10 Cs was used as a substrate (RNA10C). This molecule was completely stable in the presence of hCaf1 (Fig. 4A, lanes 5-8). A TLC assay using internally labeled RNA substrates ending with oligo $G$ sequences revealed that hCafl was also unable to degrade a substrate ending with an oligo $G$ tract (data not show). These results confirm its strong specificity toward A residues at the $3^{\prime}$ end of the substrate and its inability to act endonucleolytically. To demonstrate definitively that hCaf1 was acting exonucleolytically, we analyzed by TLC the prodwet formed in a reaction containing an RNA substrate internally labeled with $\alpha 32 \mathrm{P}$ at $\mathrm{A}$ residues. This demonstrated the exclusive release of $\alpha 32 \mathrm{P}$-AMP without the presence of di- or tri-nucleotides (Fig. 4B). This confirms a 3' exonucleolytic degradation mode.

To extend these results, we tested whether human Pop2 (hPop2), another human homolog of yeast Pop2, was also active in mRNA degradation in vitro. Interestingly, hPop2 appeared to be an extremely potent nuclease as low quantities of this recombinant factor were sufficient to fully degrade the RNA7A substrate (Fig. 5B). In comparison, only a few nucleotides from the substrate were removed by hCaf1 when the highest concentrations were compared under the same condition (Fig. 5B, note the different time scale). Consistently, only a low level of degradation intermediates could be seen. To test the specificity of the hPop2 protein, we incubated it with a non-poly(A)-substrate (Fig. 5C). Interestingly, hPop2 was able to digest this substrate completely while hCaf1 did not attack this substrate. A similar pattern of degradation was observed at various salt concentrations (see supplementary data S3). Thus, hPop2 appeared less specific for A residues than hCaf1. A TLC assay using internally labeled RNA substrates ending 


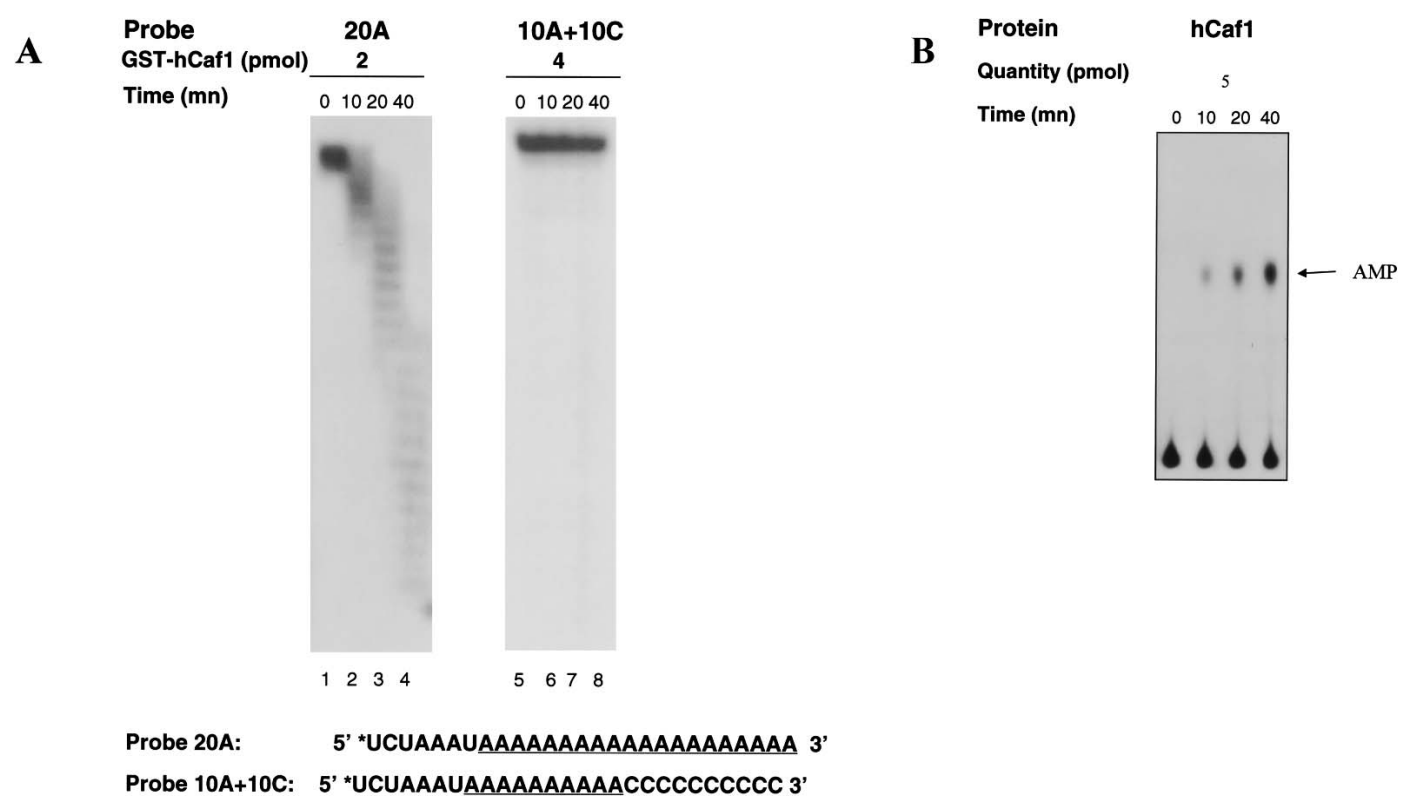

FIGURE 4. The Caf1 protein specifically degrades $3^{\prime}$ poly(A) tails. (A) Time-course reactions with the recombinant GST-hCaf1 factor and substrates ending with $20 \mathrm{~A}$ (lanes 1-4) or $10 \mathrm{~A}$ followed by $10 \mathrm{C}$ (lanes 5-8). Protein quantities are indicated. This result demonstrates that GST-hCaf1 specifically degrades 3' poly(A) tails. These data also demonstrate that GST-hCaf1 does not harbor oligo(A) specific endonuclease activity; $60 \mathrm{fmol}$ of substrate were used per reaction. (B) To confirm the exonucleolytic activity of hCafl, we also analyzed by TLC the product formed in a reaction containing hCaf1 and an RNA substrate, ending with poly(A), internally labeled with $\alpha^{32} \mathrm{P}$ at A residues. This demonstrated the exclusive release of $\alpha^{32} \mathrm{P}$-AMP (AMP marker is show by arrow) (Sigma); $100 \mathrm{fmol}$ of substrate were used per reaction.

with oligo $\mathrm{G}$ sequences revealed mononucleotide release by hPop2 indicative of exonucleolytic degradation (Fig. 5D).

We also analyzed recognition of the ribose by hCaf1 and hPop2. In this case, a single-stranded DNA oligonucleotide ending with five deoxyA residues (sequence: $5^{\prime}$-TATGT GAATTCTATGCCACCCCAAAAA-3') served as substrate. No degradation of this substrate by hCaf1 and hPop2 was detected indicating that hCaf1 and hPop2 are exclusively ribonucleases (data not shown).

\section{DISCUSSION}

The yeast Pop2/Ccr4 complex was originally described as involved in transcriptional regulation (Denis and Chen 2003). In this context, its implication in mRNA deadenylation (Daugeron et al. 2001; Tucker et al. 2001) made possible by the identification of nuclease motifs in Ccr4 and Pop2, was unexpected. It is thus not surprising that they were taken with skepticism by some (Wilusz et al. 2001). Further genetic and biochemical analyses have since then definitively confirmed a direct involvement of Pop2/Ccr4 in the deadenylation process (Chen et al. 2002; Tucker et al. 2002; Thore et al. 2003; Temme et al. 2004; Viswanathan et al. 2004). Our finding that hCaf1 and hPop2 are also nucleases supports further this conclusion. Thus, previous data showing a change in mRNA level related to Caf1 proteins function and originally thought to be the consequence of its transcriptional effect, may have to be reinterpreted as re- sulting from changes in mRNA decay. However, the clear implication of Caf1 family members in deadenylation does not exclude that they also affect transcription, as largely described in yeast and in mammals (Collart and Struhl 1994; Liu et al. 1998; Morel et al. 2003; Nakamura et al. 2004), even if direct support through run-on experiments is not available and if cognate DNA interaction site have yet to be identified.

Our results unequivocally demonstrate that human Caf1 is a $3^{\prime}-5^{\prime}$ exonuclease showing a strong, but not absolute, specificity for ribopolymers of adenine. In contrast, hPop2 appears to be less specific for poly(A). These results are consistent with those obtained with the conserved domain of the yeast Pop2 protein, which was shown to degrade preferentially, but not exclusively, poly(A) (Daugeron et al. 2001; Thore et al. 2003). The parallel between the yeast and human proteins is important if one takes into account the presence of atypical residues in the yeast Pop2 sequence making it one of the most divergent members of the Caf1 family with respect to the RNase D consensus. It is thus tempting to speculate that all members of the Pop2/Caf1 family, most of which are highly related to hCaf1 and hPop2, are endowed with deadenylase activity. This strongly suggests that they are all implicated in mRNA decay. Taken together with previously published data, our results indicate beyond reasonable doubt that, both in yeast and human, Pop2/Caf1 are active nucleases and the questions raised for the activity of yeast Pop2 can be dismissed. Interestingly, 
A

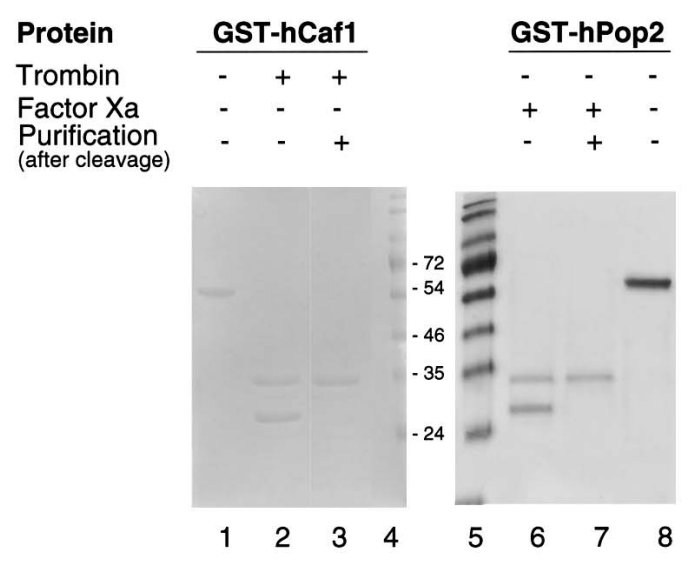

C

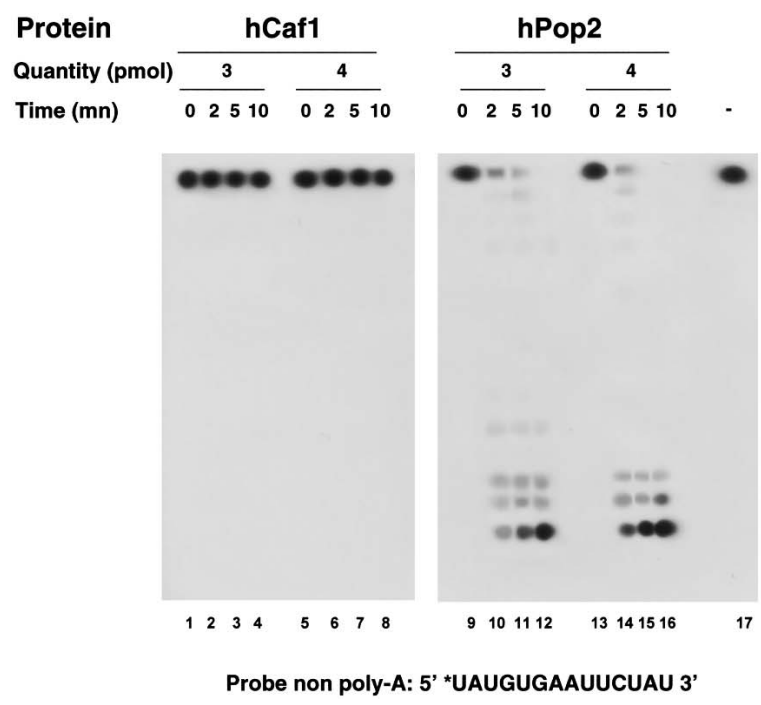

\section{B}

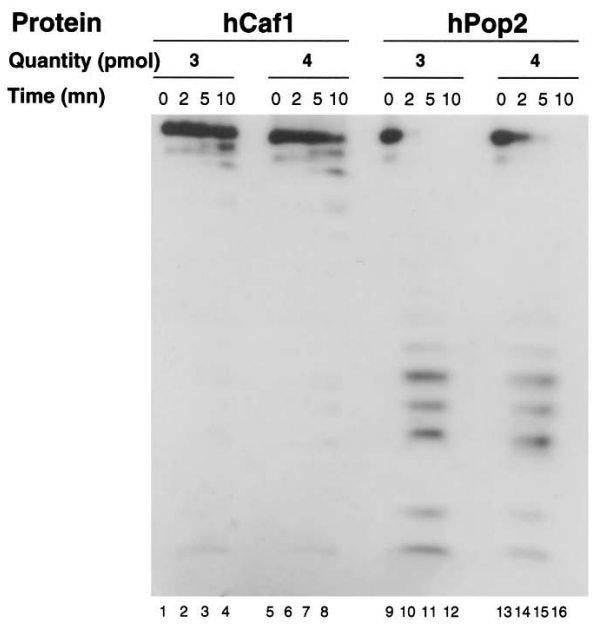

Probe RNATA : 5' *UCUAAAUAAAAAAA 3

D

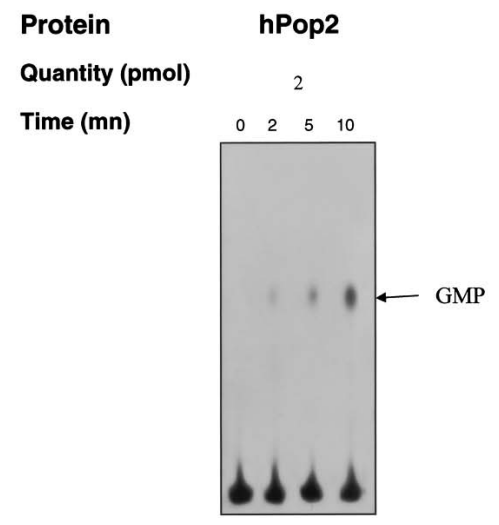

FIGURE 5. hPop2 is also a nuclease. hPop2 is a highly active RNase. (A) Purified recombinant hCaf1 and hPop2 (following purification tag removal with trombin and factor Xa, respectively) were incubated with $(B)$ a synthetic RNA oligonucleotide ending by seven A residues. Time-course reactions were performed using the indicated quantities of the recombinant factor and the products detected after fractionation on denaturing gel. Note that, given the high activity of hPop2, time-course reactions lasted only 10 min. (C) hPop2 is not highly specific for $3^{\prime}$ oligo(A) tails. hPop2 degraded efficiently a substrate lacking a 3' oligo(A) tail (lanes 9-16) while hCaf1 was unable to do so (lanes 1-8). The starting substrate was loaded in lane 17. $(D)$ To confirm the activity of hPop2, we also analyzed by TLC the product formed in a reaction containing hPop2 and an RNA substrate, ending with a poly $(\mathrm{G})$ internally labeled with $\alpha^{32} \mathrm{P}$ at $\mathrm{G}$ residues. This result shows that hPop2 is able to release $\alpha^{32} \mathrm{P}$-GMP (GMP marker is show by an arrow) (Sigma); $100 \mathrm{fmol}$ of substrate were used per reaction.

both in yeast and human, Pop2/Caf1 associates with homologs of the Ccr4 family, which are also nucleases ( $\mathrm{Du}-$ pressoir et al. 2001). One can wonder why two nucleases are required to degrade poly(A) tails. This question is even more acute in yeast where mutagenesis experiments indicated that inactivation of Pop2 does not affect cell growth (Chen et al. 2002). While this has been taken to suggest that Pop2 activity is not important for deadenylation in vivo, one cannot exclude that Pop2 deadenylase activity is re- quired under specific conditions that have not been experimentally tested. Consistent with this possibility, yeast Pop2 has been shown to be phosphorylated when cells are grown in media containing specific carbon sources (Moriya et al. 2001). Interestingly, this phosphorylation takes place in the nonconserved N-terminal domain, which is found exclusively in yeast harboring divergent catalytic site residues. It will be of interest to determine if yeast and human Pop2/ Caf1 deadenylase activities are regulated in vivo and the role 
of such regulation in the process of gene expression. In this vein, the difference in enzymatic activity that we observe between hCaf1 and hPop2 may be relevant. Indeed, the expression of one or the other of the various human homologs of yeast Pop2 may allow human cells to control gene expression by affecting the rate of mRNA deadenylation for all cellular mRNAs, or a subset of them. Further analyses of the deadenylase activity of human Caf1 and Ccr4 family members should thus provide some interesting insights in the regulation of the mRNA degradation process.

\section{MATERIAL AND METHODS}

\section{Plasmids}

Two plasmids were used to express the human Caf1 protein (hCaf1, accession number Q9UIV1). In both cases, the complete hCaf1 open reading frame, contained in a BamHI-BglII cDNA fragment, was fused in frame downstream of the GST tag of vector pGEX-2T as described (Prevot et al. 2001). In the first construct, pGEXhCAF1, the cDNA contained a $\mathrm{P}$ residue at position 4 . As this amino acid differs from the described sequence of human Caf1 (either as a result of polymorphism or mutation during cDNA synthesis), we constructed a second clone where this amino acid was changed back to A, giving plasmid pGEXhCAF1ala. pGEXhCAF1ala and the plasmid used for expression of the hCaf1 protein mutated in catalytic residues (Fig. 1) were constructed by site-directed mutagenesis using the Quick-change mutagenesis kit from Stratagene according to the manufacturer's instruction. The human Pop2 protein (hPop2, accession number AAD02685) (Fidler et al. 1999) was expressed from plasmid pGEXhPOP2 containing the full-length coding sequence of hPOP2 from pSG5hPOP2 (Prevot et al. 2001) downstream of the GST tag of the pGEX-5X vector. While hPop2 is the original name given to this factor, it was subsequently given the second name of CALIF in a latter study (Albert et al. 2000). The original nomenclature is used throughout this manuscript.

\section{Protein preparations}

Proteins were expressed in E. coli strains BL21CodonPlus or Rosetta (DE3) (Novagen). Expression conditions were essentially as described previously (Daugeron et al. 2001). Cell pellets from 500 $\mathrm{mL}$ cultures were lyzed by sonication in PBS. Cellular debris and a fraction of the recombinant factor that was insoluble probably due to poor folding in the absence of natural partners were removed by centrifugation at $17,000 \mathrm{~g}$ for $30 \mathrm{~min}$. The supernatant was mixed in a $15 \mathrm{~mL}$ Falcon tube with $0.4 \mathrm{~mL}$ of a $50 \%$ slurry of glutathione agarose beads previously equilibrated in PBS. The closed tube was rotated at $4^{\circ} \mathrm{C}$ for $1 \mathrm{~h}$. Beads were transferred to an Econo column (Biorad) and washed with 50 column volumes of PBS at $4^{\circ} \mathrm{C}$. The purified protein was recovered by elution with $10 \mathrm{mM}$ reduced glutathione in $50 \mathrm{mM}$ Tris $\mathrm{HCl}$ pH8.0. hCAF1 and hPOP2 proteins were also purified following cleavage with thrombin or factor-X to release them from the column-bound GST fusions. SDS/PAGE and Coomassie staining were used to confirm the integrity of the full-length fusion proteins (Figs. 2A, $5 \mathrm{~A})$.
Recombinant factors were stored, after dialysis, in PBS containing 50\% glycerol. Protein concentration was determined using the Protein Assay Reagent (Biorad).

\section{Preparation of substrates and in vitro deadenylation assay}

The commercially synthesized RNA and ssDNA oligonucleotides used as substrates $(10 \mathrm{pmol})$ were labeled with T4 polynucleotide kinase and $[\gamma-32 \mathrm{P}]$ ATP. After purification on Sephadex G-25 spin columns, labeled substrates, were diluted and normalized to $10,000 \mathrm{cpm} / \mu \mathrm{L}(60-100 \mathrm{fmol}$ of RNA substrates) (equivalent to 6-10 nM).

The following RNA and ssDNA oligonucleotides were employed:

RNA7A: $5^{\prime}$ UCUAAAU $(A)_{7} 3^{\prime}$;

RNA20A: 5' UCUAAAU(A) ${ }_{20} 3^{\prime}$;

RNA5A: $5^{\prime}$-UAUGUGAAUUCUAUGCCACCCC $(\mathrm{A})_{5}-3^{\prime}$;

RNA10C: $5^{\prime}$-UCUAAAU $(\mathrm{A})_{10}(\mathrm{C})_{10} \mathbf{- 3}^{\prime}$;

RNAr: 5'-UAUGUGAAUUCUAU-3'; and

DNA5A: 5'-TATGTGAATTCTATGCCACCCC $(\mathrm{A})_{5}-3^{\prime}$.

Nuclease assays involving bulk-labeled poly(A) degradation followed by quantification of released nucleotides as TCA soluble material were performed as described previously (Daugeron et al. 2001). Synthetic RNA or DNA oligonucleotides labeled at their $5^{\prime}$ end and in vitro transcribed RNA labeled with radioactive $\alpha \mathrm{UTP}^{32}$ were used to assay for the degradation of substrates of defined sequence. Degradation reactions contained $10 \mathrm{mM}$ Tris- $\mathrm{HCl}$ pH8.0, 1 mM MgOAc, 2 mM DTT, 0.02\% NP40, 2 mM spermidine. Reactions, initiated by addition of indicated amounts of recombinant protein, were incubated at $30^{\circ} \mathrm{C}$ for the indicated times. After addition of formamide, reaction products were fractionated on appropriate $8 \%-15 \%$ acrylamide urea gels and visualized by autoradiography or exposition to PhosphorImager screens.

\section{Exonucleolytic release of mononucleotides}

Uniformly labeled RNA was transcribed in vitro transcription with T3 polymerase (Promega). Plasmids pT3L3(A30) and pT3ML43(G30) (Astrom et al. 1991), digested respectively with Nsil and Apa1, were used as template. pT3L3(A30) transcription was performed in presence of $\left[\alpha-{ }^{32} \mathrm{P}\right]$ ATP while linearized pT3ML43(G13) was transcribed in presence of $\left[\alpha^{32} \mathrm{P}\right]$ GTP; 100 fmol of the resulting RNAs were used to assay for the degradation of substrates of defined sequence as it was described above. Reactions products were stopped by addition of $0.5 \mathrm{M}$ of EDTA and products fractionated by PEI-cellulose thin-layer chromatography developed in $0.45 \mathrm{M}$ (NH4)2SO4 and detected by autoradiography.

\section{SUPPLEMENTARY MATERIAL}

Supplementary material can be available upon request (send an e-mail message containing the keyword "CAF1/POP2 supplementary data" to the corresponding authors). 


\section{ACKNOWLEDGMENTS}

We thank A. Virtanen for the gift of plasmids and B. Krop for assistance. The work in L. Corbo laboratory was supported by the "Association pour la Recherche sur le Cancer," (ARC), the INSERM, the Ligue Nationale Contre le Cancer, Comité du Rhône, and Comité de la Drôme. C. Bianchin holds a doctoral fellowship from the Ligue Nationale Contre le Cancer (Comité de la Saône et Loire). In the group of B. Séraphin, this work was supported by "La Ligue contre le cancer" (Equipe labellisée 2001), the CNRS, and the Ministry for Research (ACI BCMS).

Received July 16, 2004; accepted December 29, 2004.

\section{REFERENCES}

Albert, T.K., Lemaire, M., van Berkum, N.L., Gentz, R., Collart, M.A., and Timmers, H.T. 2000. Isolation and characterization of human orthologs of yeast CCR4-NOT complex subunits. Nucleic Acids Res. 28: 809-817.

Astrom, J., Astrom, A., and Virtanen, A. 1991. In vitro deadenylation of mammalian mRNA by a HeLa cell 3 ' exonuclease, EMBO J. 10: 3067-3071.

Boeck, R., Tarun Jr., S., Rieger, M., Deardorff, J.A., Muller-Auer, S., and Sachs, A.B. 1996. The yeast Pan2 protein is required for poly(A)-binding protein-stimulated poly(A)-nuclease activity. $J$. Biol. Chem. 271: 432-438.

Brown, C.E., Tarun Jr., S.Z., Boeck, R., and Sachs, A.B. 1996. PAN3 encodes a subunit of the Pablp-dependent poly(A) nuclease in Saccharomyces cerevisiae. Mol. Cell Biol. 16: 5744-5753.

Chen, J., Chiang, Y.C., and Denis, C.L. 2002. CCR4, a 3'-5' poly(A) RNA and ssDNA exonuclease, is the catalytic component of the cytoplasmic deadenylase. EMBO J. 21: 1414-1426.

Collart, M.A. and Struhl, K. 1994. NOT1(CDC39), NOT2(CDC36), NOT3, and NOT4 encode a global-negative regulator of transcription that differentially affects TATA-element utilization. Genes \& Dev. 8: 525-537.

Daugeron, M.C., Mauxion, F., and Seraphin, B. 2001. The yeast POP2 gene encodes a nuclease involved in mRNA deadenylation. Nucleic Acids Res. 29: 2448-2455.

Dehlin, E., Wormington, M., Korner, C.G., and Wahle, E. 2000. Capdependent deadenylation of mRNA. EMBO J. 19: 1079-1086.

Denis, C.L. and Chen, J. 2003. The CCR4-NOT complex plays diverse roles in mRNA metabolism. Prog. Nucleic Acid Res. Mol. Biol. 73: $221-250$.

Dlakic, M. 2000. Functionally unrelated signalling proteins contain a fold similar to $\mathrm{Mg} 2+-$ dependent endonucleases. Trends Biochem. Sci. 25: 272-273.

Dupressoir, A., Morel, A.P., Berbot, W., Loireau, M.-P., Corbo, L., and Heidmann, T. 2001. Identification of four families of yCCR4- and $\mathrm{Mg} 2+$-dependent endonuclease-related proteins in higher eukaryotes, and characterization of orthologs of yCCR 4 with a conserved leucine-rich repeat essential for hCAF1/hPOP2 binding. BMC Genomics 2: 9.

Fidler, C., Wainscoat, J.S., and Boultwood, J. 1999. The human POP2 gene: Identification, sequencing, and mapping to the critical region of the 5q-syndrome. Genomics 56: 134-136.

Gao, M., Fritz, D.T., Ford, L.P., and Wilusz, J. 2000. Interaction between a poly(A)-specific ribonuclease and the $5^{\prime}$ cap influences mRNA deadenylation rates in vitro. Mol. Cell 5: 479-488.

Korner, C.G., Wormington, M., Muckenthaler, M., Schneider, S., Dehlin, E., and Wahle, E. 1998. The deadenylating nuclease (DAN) is involved in poly(A) tail removal during the meiotic maturation of Xenopus oocytes. EMBO J. 17: 5427-5437.

Liu, H.Y., Badarinarayana, V., Audino, D.C., Rappsilber, J., Mann, M., and Denis, C.L. 1998. The NOT proteins are part of the CCR4 transcriptional complex and affect gene expression both positively and negatively. EMBO J. 17: 1096-1106.

Lykke-Andersen, J. 2002. Identification of a human decapping complex associated with hUpf proteins in nonsense-mediated decay. Mol. Cell. Biol. 22: 8114-8121.

Morel, A.P., Sentis, S., Bianchin, C., Le Romancer, M., Jonard, L., Rostan, M.C., Rimokh, R., and Corbo, L. 2003. BTG2 antiproliferative protein interacts with the human CCR4 complex existing in vivo in three cell-cycle-regulated forms. J. Cell Sci. 116: 29292936.

Moriya, H., Shimizu-Yoshida, Y., Omori, A., Iwashita, S., Katoh, M., and Sakai, A. 2001. Yak1p, a DYRK family kinase, translocates to the nucleus and phosphorylates yeast Pop2p in response to a glucose signal. Genes \& Dev. 15: 1217-1228.

Muhlrad, D., Decker, C.J., and Parker, R. 1994. Deadenylation of the unstable mRNA encoded by the yeast MFA2 gene leads to decapping followed by $5^{\prime} \rightarrow 3^{\prime}$ digestion of the transcript. Genes \& Dev. 8: 855-866.

. 1995. Turnover mechanisms of the stable yeast PGK1 mRNA. Mol. Cell. Biol. 15: 2145-2156.

Nakamura, T., Yao, R., Ogawa, T., Suzuki, T., Ito, C., Tsunekawa, N., Inoue, K., Ajima, R., Miyasaka, T., Yoshida, Y., et al. 2004. Oligoastheno-teratozoospermia in mice lacking Cnot7, a regulator of retinoid X receptor beta. Nat. Genet. 36: 528-533.

Parker, R. and Song, H. 2004. The enzymes and control of eukaryotic mRNA turnover. Nat. Struct. Mol. Biol. 11: 121-127.

Prevot, D., Morel, A.P., Voeltzel, T., Rostan, M.C., Rimokh, R., Magaud, J.P., and Corbo, L. 2001. Relationships of the antiproliferative proteins BTG1 and BTG2 with CAF1, the human homolog of a component of the yeast CCR4 transcriptional complex: Involvement in estrogen receptor alpha signaling pathway. J. Biol. Chem. 276: 9640-9648.

Temme, C., Zaessinger, S., Meyer, S., Simonelig, M., and Wahle, E. 2004. A complex containing the CCR4 and CAF1 proteins is involved in mRNA deadenylation in Drosophila. EMBO J. 23: 2862 2871.

Thompson, J.D., Gibson, T.J., Plewniak, F., Jeanmougin, F., and Higgins, D.G. 1997. The CLUSTAL_X windows interface: Flexible strategies for multiple sequence alignment aided by quality analysis tools. Nucleic Acids Res. 25: 4876-4882.

Thore, S., Mauxion, F., Seraphin, B., and Suck, D. 2003. X-ray structure and activity of the yeast Pop2 protein: A nuclease subunit of the mRNA deadenylase complex. EMBO Rep. 4: 1150-1155.

Tucker, M., Valencia-Sanchez, M.A., Staples, R.R., Chen, J., Denis, C.L., and Parker, R. 2001. The transcription factor associated Ccr4 and Caf1 proteins are components of the major cytoplasmic mRNA deadenylase in Saccharomyces cerevisiae. Cell 104: 377-386.

Tucker, M., Staples, R.R., Valencia-Sanchez, M.A., Muhlrad, D., and Parker, R. 2002. Ccr4p is the catalytic subunit of a Ccr4p/Pop2p/ Notp mRNA deadenylase complex in Saccharomyces cerevisiae. EMBO J. 21: 1427-1436.

Van Dijk, E., Cougot, N., Meyer, S., Babajko, S., Wahle, E., and Seraphin, B. 2002. Human Dcp2: A catalytically active mRNA decapping enzyme located in specific cytoplasmic structures. $E M B O$ J. 21: 6915-6924.

Van Dijk, E., Le Hir, H., and Seraphin, B. 2003. DcpS can act in the $5^{\prime}-3^{\prime}$ mRNA decay pathway in addition to the $3^{\prime}-5^{\prime}$ pathway. Proc. Natl. Acad. Sci. 100: 12081-12086.

Viswanathan, P., Ohn, T., Chiang, Y.C., Chen, J., and Denis, C.L. 2004. Mouse CAF1 can function as a processive deadenylase/ $3^{\prime}-$ $5^{\prime}$-exonuclease in vitro but in yeast the deadenylase function of CAF1 is not required for mRNA poly(A) removal. J. Biol. Chem. 279: 23988-23995.

Wang, Z. and Kiledjian, M. 2001. Functional link between the mammalian exosome and mRNA decapping. Cell 107: 751-762.

Wang, Z., Jiao, X., Carr-Schmid, A., and Kiledjian, M. 2002. The $\mathrm{hDcp} 2$ protein is a mammalian mRNA decapping enzyme. Proc. Natl. Acad. Sci. 99: 12663-12668.

Wilusz, C.J., Wormington, M., and Peltz, S.W. 2001. The cap-to-tail guide to mRNA turnover. Nat. Rev. Mol. Cell. Biol. 2: 237-246. 

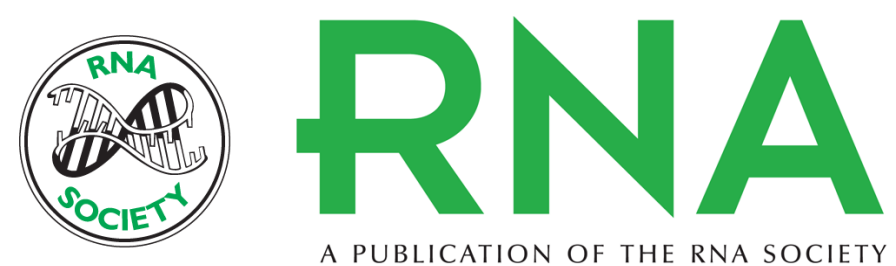

A PUBLICATION OF THE RNA SOCIETY

\section{Conservation of the deadenylase activity of proteins of the Caf1 family in human}

CLAIRE BIANCHIN, FABIENNE MAUXION, STÉPHANIE SENTIS, et al.

RNA 2005 11: 487-494

\section{References This article cites 34 articles, 18 of which can be accessed free at: http://rnajournal.cshlp.org/content/11/4/487.full.html\#ref-list-1}

\section{License}

Email Alerting Receive free email alerts when new articles cite this article - sign up in the box at the Service top right corner of the article or click here.

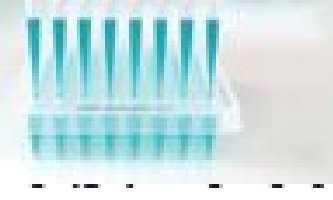

